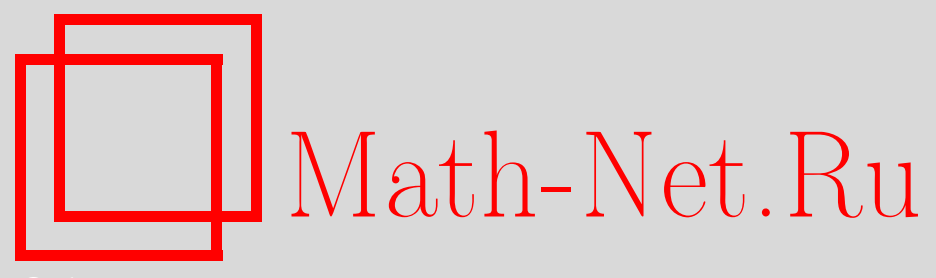

С. А. Клоков, О нижних оценках скорости перемешивания для одного класса марковских процессов, Теория вероятн. и ее примен., 2006, том 51, выпуск 3, 600-607

DOI: https://doi.org/10.4213/tvp42

Использование Общероссийского математического портала Math-Net.Ru подразумевает, что вы прочитали и согласны с пользовательским соглашением

http: //www . mathnet.ru/rus/agreement

Параметры загрузки:

IP : 3.85 .73 .92

26 апреля 2023 г., 16:42:44

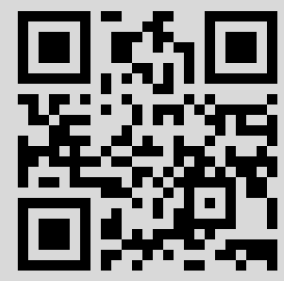


науки и техники, сер. соврем. пробл. матем., фундам. напр., т. 46. М.: ВИНИТИ, 1989.

23. Эллиотт P. Стохастический анализ и его приложения. М.: Мир, 1986, $351 \mathrm{c.}$

24. Анулова С. В., Веретенников А.Ю., Крылов Н. В., Липчер Р. Ш., Ширяев А.Н. Стохастическое исчисление. Итоги науки и техники, сер. соврем. пробл. матем., фундам. напр., т. 45. М.: ВИНИТИ, 1989.

25. Фридман $A$. Уравнения с частными производными параболического типа. М.: Мир, 1968, 427 c.

Поступила в редакцию 30.IV.2004

(C) $2006 \mathrm{r}$.

КЛОКОВ С. А. ${ }^{*}$

\section{О НИЖНИХ ОЦЕНКАХ СКОРОСТИ ПЕРЕМЕШИВАНИЯ ДЛЯ ОДНОГО КЛАССА МАРКОВСКИХ ПРОЦЕССОВ ${ }^{1)}$}

В работе установлены нижние оценки скорости $\alpha$-перемешивания для некоторых моделей из одного класса стационарных марковских процессов при выполнении условий возвратности. Дано сравнение с известными верхними оценками скорости $\beta$-перемешивания для этого же класса процессов в случаях полиномиальной и субэкспоненциальной сходимости. В последнем случае показано, что оценки являются в определенном смысле неулучшаемыми.

Ключевые слова и фразы: марковские процессы, возвратность, инвариантная мера, коэффициенты перемешивания, полиномиальная скорость, субэкспоненциальная скорость.

1. Введение. Пусть $\left(X_{n}\right)$ - случайный процесс со значениями в $\mathbf{R}^{d}$. Обозначим через $\mathscr{F} \leqslant n=\sigma\left\{X_{j}: j \leqslant n\right\}$ и $\mathscr{F}_{\geqslant n}=\sigma\left\{X_{j}: j \geqslant n\right\}$ «прошлое» и «будущее» этого процесса. Существует несколько способов определить понятие слабой зависимости между $\sigma$-алгебрами $\mathscr{F} \leqslant m$ и $\mathscr{F} \geqslant m+n$ при $n \rightarrow \infty$. Рассмотрим следуюшие три коэффициента перемешивания, введенные в 50-60-х годах М. Розенблаттом [14], А. Н. Колмогоровым и И. А. Ибрагимовым [9]:

$$
\begin{aligned}
\alpha(n) & =\sup _{m \geqslant 0} \sup _{A \in \mathscr{F} \leqslant m}, B \in \mathscr{F} \geqslant m+n \\
\beta(n) & =\sup _{m \geqslant 0} \mathbf{E} \operatorname{var}_{B \in \mathscr{F} \geqslant m+n}(\mathbf{P}(B \mid \mathscr{F} \leqslant m)-\mathbf{P}(B)), \\
\varphi(n) & =\sup _{m \geqslant 0} \sup _{A \in \mathscr{F} \leqslant m}, B \in \mathscr{F} \geqslant m+\mathbf{P}(B) \mid, \\
& \mid \mathbf{P}(A)>0
\end{aligned}
$$

где символ var обозначает полную вариацию заряда (меры со знаком).

Если $\alpha(n) \rightarrow 0$ при $n \rightarrow \infty$, то говорят, что имеет место $\alpha$-перемешивание (или сильное перемешивание). Аналогично вводятся $\beta$-перемешивание и $\varphi$-перемешивание (или равномерно сильное перемешивание). Хорошо известны неравенства между этими коэффициентами:

$$
\alpha(n) \leqslant \beta(n) \leqslant \varphi(n),
$$

* Омский филиал Института математики им. С.Л. Соболева СО РАН, ул. Певцова, 13, 644099 Омск, Россия; School of Mathematics, University of Leeds, Leeds LS2 9JT, UK; e-mail: klokov@iitam.omsk.net.ru

1) Работа выполнена при поддержке грантов EPSRC GR/R40746/01 (UK), NFGRF 2301863 (University of Kansas) и РФФИ 03-01-00045. 
показывающие, что $\alpha$-перемешивание является наиболее слабым, а $\varphi$-перемешивание - самым сильным из них.

Доказательство того, что стохастические модели обладают свойством перемешивания, важно, поскольку многие результаты из области предельных теорем для слабо зависимых величин и области оценки параметров опираются на предположения о перемешивании и, в частности, на его скорость. Поскольку дать полный библиографический список в этой статье не представляется возможным, мы отсылаем читателя к работам [7]-[9], [12] и спискам литературы в них.

Примеры процессов с перемешиванием получены И.А. Ибрагимовым [9], Ю. А. Давыдовым [5], Р. Брэдли [1]-[4], А. Ю. Веретенниковым [15], [16], М. Н. Малышкиным [11], автором и А. Ю. Веретенниковым [10], С. П. Майном и Р. Л. Твиди [13] и др. Однако степень изученности вопроса о классах случайных процессов, удовлетворяющих тому или иному условию перемешивания, представляется недостаточной.

Результат статьи [5] показывает, что в классе марковских процессов с $\varphi$-перемешиванием зависимость либо убывает экспоненциально быстро, либо не убывает совсем в том смысле, что $\varphi(n) \equiv 1$. Во многих важных случаях $\varphi$-перемешивание отсутствует. Здесь был бы полезен более слабый тип зависимости вроде $\alpha$ - или $\beta$-перемешивания.

В работах [16], [10] рассмотрен один класс марковских процессов при условиях возвратности (описание модели дается в следуюшем пункте; см. также [15], [16], [11], где вводится аналогичная модель с непрерывным временем) и получены результаты о скорости $\beta$-перемешивания. В настоящей статье вычислены нижние оценки коэффициента $\alpha$-перемешивания для некоторых представителей вышеупомянутого класса процессов. Используя неравенства (4) и верхние оценки для $\beta$-перемешивания из [16], $[10]$, можно судить о точности полученных результатов.

Оставшаяся часть статьи имеет следующую структуру. В п. 2 дается описание модели и формулируются известные результаты, относящиеся к ней. В пп. 3 и 4 доказаны нижние оценки для скорости $\alpha$-перемешивания (а следовательно, и $\beta$-перемешивания) для стационарной версии процесса и сделаны выводы.

2. Описание модели и известные результаты. Следуя [16], [10], рассмотрим класс марковских цепей с фазовым пространством $\mathbf{R}^{d}$, которые могут быть представлены в форме нелинейной авторегрессии:

$$
X_{n+1}=g\left(X_{n}\right)+V_{n+1},
$$

где $\left(V_{n}\right)$ - это последовательность независимых одинаково распределенных случайных величин с нулевыми средними, а функция $g: \mathbf{R}^{d} \rightarrow \mathbf{R}^{d}$ удовлетворяет оценкам

$$
|g(x)| \leqslant \begin{cases}|x|-r|x|^{-p}, & |x|>R_{0} \\ C_{0}, & |x| \leqslant R_{0}\end{cases}
$$

с некоторым $p \in[0,1]$. Это условие может быть интерпретировано следующим образом. Вне шара $B_{R_{0}}=\left\{x:|x| \leqslant R_{0}\right\}$ функция $g$ обеспечивает «притяжение» к началу координат. Параметры $p$ и $r$ регулируют силу этого притяжения. Внутри шара $B_{R_{0}}$ функция $g$ предполагается ограниченной.

Напомним определение локального условия Дёблина (см. [16]). Пусть $B$ является компактным множеством, $\tau_{1}=\inf \left\{n \geqslant 0: X_{n} \in B\right\}, \tau_{k+1}=\inf \left\{n>\tau_{k}: X_{n} \in B\right\}$. Определим «процесс на $B \gg, X_{n}^{B}=X_{\tau_{n}}$, и обозначим его переходную функцию за $n$ шагов через $P^{B}(n, x, d y)$. Говорят, что выполнено локальное условие Дёблина, если для каждого достаточно большого $R$ и $B=\left\{x \in \mathbf{R}^{d}:|x| \leq R\right\}$

$$
\inf _{x, x^{\prime} \in B} \int_{\mathbf{R}^{d}} \min \left\{1, \frac{P^{B}(n, x, d y)}{P^{B}\left(n, x^{\prime}, d y\right)}\right\} P^{B}\left(n, x^{\prime}, d y\right)=q(R)>0,
$$

где $P^{B}(n, x, d y) / P^{B}\left(n, x^{\prime}, d y\right)$ означает производную абсолютно непрерывной компоненты меры $P^{B}(n, x, d y)$ по отношению к мере $P^{B}\left(n, x^{\prime}, d y\right)$. Сингулярная часть может быть ненулевой. Условие $\left(D_{l}\right)$ обеспечивает отсутствие сингулярности мер внутри шара $B$. 
Всюду в данной работе на функцию $g$ и последовательность $\left(V_{n}\right)$ будут накладываться ограничения, гарантирующие существование и единственность инвариантной вероятностной меры, т.е. выполнение некоторого дополнительного свойства эргодичности, обычно называемого положительностью.

Условия типа $\left(D_{l}\right)$ часто используются в теории марковских цепей и обобщают классическое условие Дёблина [6]. Если последнее выполнено для всего фазового пространства, то скорость сходимости и скорость перемешивания экспоненциальны.

При некоторых предположениях на существование моментов у распределения случайных величин $\left(V_{n}\right)$ получены следующие два результата о верхних оценках для скорости сходимости к инвариантной мере и $\beta$-перемешивания (заметим, что все маргинальные распределения, коэффициенты перемешивания и скорости сходимости зависят от начального значения $X_{0}=x$ и нижний индекс $x$ отражает это).

Предложение 1 (см. [16, теоремы 1-3]). Пусть прочесс $\left(X_{n}\right)$ удовлетворяет (5) u (6) с параметрами $p=1$ и достаточно большим $r, \mathbf{E}\left|V_{n}\right|^{m_{0}}<\infty, m_{0}>4, u$ выполнено локальное условие Дёблина $\left(D_{l}\right)$. Тогда существует единственная инвариантная вероятностная мера $\mu_{\mathrm{inv}}$, обладающая абсолютными моментами порядков не менее чем $m_{0}-2, u$

$$
\begin{aligned}
\operatorname{var}\left(\mu_{n, x}-\mu_{\text {inv }}\right) & \leqslant C\left(1+|x|^{m}\right) n^{-(1+k)}, \\
\beta_{x}(n) & \leqslant C\left(1+|x|^{m}\right) n^{-(1+k)},
\end{aligned}
$$

где $m \in\left(2, m_{0}-2\right)$ u $k<(m-2) / 2$ могут быть выбраны произвольно, а $C$ зависит om $m u k$.

Пусть теперь параметр $p$ принадлежит открытому интервалу $(0,1)$, что обеспечивает более сильное притяжение к началу координат. Предположим также, что случайные величины $\left(V_{n}\right)$ обладают конечными субэкспоненциальными моментами. В этих более ограничительных предположениях получена лучшая скорость сходимости.

Предложение 2 (см. [10, теорема 1]). Пусть прочесс $\left(X_{n}\right)$ удовлетворяет условиям (5) $u$ (6) с параметрами $p \in(0,1) u r>0$ и существуют $K>0 u$ $\alpha \in(0,1-p]$ тажие, что $\mathbf{E} \exp \left\{k\left|V_{n}\right|^{\alpha}\right\}<\infty$ для $0 \leqslant k<K$. Предположим тажже, что выполнено локальное условие Дёблина $\left(D_{l}\right)$. Тогда существует единственная инвариантная вероятностная мера $\mu_{\mathrm{inv}} u$

$$
\begin{aligned}
\operatorname{var}\left(\mu_{n, x}-\mu_{\mathrm{inv}}\right) & \leqslant C(x) \exp \left\{-c n^{\delta}\right\}, \\
\beta_{x}(n) & \leqslant C(x) \exp \left\{-c n^{\delta}\right\}
\end{aligned}
$$

$c \delta \in(0, \alpha /(1+p))$, некоторой положительной функиией $C(x)$ и константой $c>0$, которые зависят от $\delta$.

3. Полиномиальная оценка. Идея получения нижних оценок для $\alpha(n)$ такова. Рассмотрим конкретную одномерную стационарную стохастическую модель $\left(X_{n}\right)$, $n \in \mathbf{Z}$, предъявим некоторые события $A \in \mathscr{F}_{\leqslant 0}^{X}, B \in \mathscr{F}_{\geqslant n}^{X}, \mathbf{P}(A)>0$, и вычислим нижнюю оценку для $\mathbf{P}(A)(\mathbf{P}(B \mid A)-\mathbf{P}(B))$. При этом последовательность шума $\left(V_{n}\right)$ выбрана так, что величины имеют полиномиальные или субэкспоненциальные «хвосты», а функция $g$ обеспечивает как можно более слабое притяжение к началу координат. Такой подход, по-видимому, должен привести к нижней оценке, которая близка к оптимальной.

Теорема 1. Для каждого $\varepsilon>0$ существует стационарная стохастическая модель, удовлетворяющая предположениям предложения 1 , для которой

$$
C_{1} n^{-\left(m_{0}+\varepsilon\right) / 2} \leqslant \alpha(n) \leqslant \beta(n) \leqslant C_{2} n^{-\left(m_{0}-\varepsilon-2\right) / 2}, \quad C_{1}, C_{2}>0 .
$$

Д о к а з а т е л ь с т в о. Верхняя граница доказана в предложении 1 , так что необходимо обосновать лишь нижнюю границу.

Рассмотрим случай $d=1$ и положим

$$
g(x)= \begin{cases}0, & x \leqslant R_{0} \\ x-r x^{-1}, & x>R_{0}\end{cases}
$$


где $r>0$ и $R_{0}>0$ имеют тот же смысл, что и в (6). Предположим, что распределение случайных величин $V_{n}$ задается с помошью соотношения

$$
\mathbf{P}\left\{V_{n} \in A\right\}=\frac{1}{2} \mathbf{1}_{A}\left(-\frac{\gamma}{\gamma-1}\right)+\frac{\gamma}{2} \int_{A \cap[1, \infty)} \frac{d x}{x^{\gamma+1}},
$$

где $1_{A}$ обозначает индикатор множества $A$ и $\gamma>2$ является параметром. Элементарные вычисления показывают, что $\mathbf{E} V_{n}=0$ и $\mathbf{E}\left|V_{n}\right|^{m}<\infty$ для любого $m<\gamma$, но $\mathbf{E}\left|V_{n}\right|^{\gamma}=\infty$

Покажем, что марковская цепь, заданная (5), (11) и (12) для $\gamma>4$, обладает инвариантной вероятностной мерой. Это будет следовать из предложения 1 , если только выполнено локальное условие Дёблина. Последнее будет иметь место, если мы покажем, что, стартуя из любых двух различных точек $x, x^{\prime} \in[-R, R], R \geqslant R_{0}$, две копии цепи $\left(X_{n}\right)$ и $\left(X_{n}^{\prime}\right)$, управляемые одной и той же последовательностью шума $\left(V_{n}\right)$, с ненулевой вероятностью попадают одновременно в одну точку фазового пространства за конечный промежуток времени. Рассмотрим траекторию, когда случайные величины из последовательности шума $\left(V_{n}\right)$ принимают только значения $-\gamma /(\gamma-1)$. После конечного числа шагов, скажем $n_{x}$, снос приведет процесс $X_{n}$ в начало координат, и затем он попадет в состояние $-\gamma /(\gamma-1)$. Вероятность этого события равна $2^{-n_{x}}$. Цепь останется в этой точке на протяжении $k$ дополнительных шагов с (условной) вероятностью $2^{-k}$. Таким образом, процессы $\left(X_{n}\right)$ и $\left(X_{n}^{\prime}\right)$ после $n_{x} \vee n_{x^{\prime}}$ шагов оказываются в состоянии $-\gamma /(\gamma-1)$ с вероятностью не меньшей, чем $2^{-\left(n_{\boldsymbol{x}} \vee n_{\boldsymbol{x}^{\prime}}\right)}$, и локальное условие Дёблина выполнено.

Изучим некоторые свойства инвариантной меры $\mu_{\mathrm{inv}}$. Носитель $\mu_{\mathrm{inv}}$ - это подмножество $[-\gamma /(\gamma-1), \infty)$ в силу (11) и (12). Точка $-\gamma /(\gamma-1)$ является атомом для $\mu_{\mathrm{inv}}$. В самом деле, возьмем какой-нибудь интервал $[A, B]$ с $\mu_{\mathrm{inv}}[A, B]=\mu>0$. После конечного числа шагов, скажем $n_{\mu}$, снос и значения шума, равные $-\gamma /(\gamma-1)$, перемешают все точки интервала $[A, B]$ в точку $-\gamma /(\gamma-1)$ с вероятностью $2^{-n_{\mu}}$. Обозначим $\delta=\mu_{\mathrm{inv}}\{-\gamma /(\gamma-1)\}>0$.

Пусть $\gamma>4, m_{0}=\gamma-\varepsilon$ (см. предложение 1 и (12)). Рассмотрим следующие события для стационарной версии процесса $\left(X_{n}\right)$ :

$$
A_{n}=\left\{X_{0}>M \sqrt{n \ln \ln n}\right\}, \quad B_{n}=\left\{X_{n}>\frac{1}{2} M \sqrt{n \ln \ln n}\right\}, \quad M>0 .
$$

Окончательное значение $M$ будет выбрано позже. Так как $\mu_{\mathrm{inv}}$ является вероятностной мерой, имеем:

$$
\mathbf{P}\left(A_{n}\right)=\mu_{\mathrm{inv}}(M \sqrt{n \ln \ln n},+\infty) \longrightarrow 0, \quad n \rightarrow \infty,
$$

и, согласно (12),

$$
\begin{aligned}
\mathbf{P}\left(A_{n}\right) & \geqslant \mathbf{P}\left\{X_{-1}=-\gamma(\gamma-1)^{-1}, X_{0}>M \sqrt{n \ln \ln n}\right\} \\
& =\delta \mathbf{P}\left\{X_{0}>M \sqrt{n \ln \ln n} \mid X_{-1}=-\gamma(\gamma-1)^{-1}\right\} \\
& =\delta \mathbf{P}\left\{V_{0}>M \sqrt{n \ln \ln n}+\gamma(\gamma-1)^{-1}\right\} \geqslant c(M \sqrt{n \ln \ln n})^{-\gamma}
\end{aligned}
$$

с некоторой константой $c>0$. Очевидно, что аналогичные соотношения верны и для $\mathbf{P}\left(B_{n}\right)$.

Оценим $\mathbf{P}\left(B_{n} \mid A_{n}\right)$ снизу. Применяя закон повторного логарифма для суммы случайных величин $\left(V_{n}\right)$, выберем константу $C>0$ так, чтобы событие

$$
D=\bigcap_{k \geqslant 3}\left\{\sum_{j=1}^{k} V_{j} \geqslant-C \sqrt{k \ln \ln k}\right\}
$$

имело положительную вероятность. Это означает, что существует достаточно много траекторий случайного блуждания $\sum_{j=1}^{k} V_{j}$, которые целиком лежат выше уровня $-C \sqrt{k \ln \ln k}$. 
Лемма 1. Пусть $n$ достаточно велико и произошли события $A_{n}$ u $D$. Tогда для всех $1 \leqslant k \leqslant n$ события

$$
\begin{array}{cl}
\left\{X_{k} \geqslant M \sqrt{n \ln \ln n}-2 k\right\}, & k=1,2, \\
\left\{X_{k} \geqslant M \sqrt{n \ln \ln n}-2 C \sqrt{k \ln \ln k}\right\}, & k \geqslant 3,
\end{array}
$$

тажже произошли.

Д ок а з а т е л ь с т в о. Так как $\gamma>4, V_{j} \geqslant-\gamma /(\gamma-1)$ и $n$ велико, то

$$
X_{1}=X_{0}-\frac{r}{X_{0}}+V_{1} \geqslant X_{0}-2
$$

и, аналогично, $X_{2} \geqslant X_{0}-4, X_{3} \geqslant X_{0}-6$. Увеличивая $C$ в случае необходимости, имеем:

$$
X_{0}-6 \geqslant M \sqrt{n \ln \ln n}-2 C \sqrt{3 \ln \ln 3},
$$

и событие для $k=3$ произошло.

Используя индукцию, докажем, что требуемое событие для $X_{k+1}$ произошло, если все предыдущие события для $X_{1}, \ldots, X_{k}$ также произошли. В силу (11) можно представить $X_{k+1}$ в виде

$$
X_{k+1}=X_{0}+\sum_{j=1}^{k+1} V_{j}-r \sum_{j=0}^{k} \frac{1}{X_{j}}
$$

Запишем оценки:

$$
\begin{gathered}
\sum_{j=1}^{k+1} V_{j} \geqslant-C \sqrt{(k+1) \ln \ln (k+1)} \\
r \sum_{j=0}^{k} \frac{1}{X_{j}} \leqslant \frac{r(k+1)}{M \sqrt{n \ln \ln n}-2 C \sqrt{k \ln \ln k}} \leqslant \frac{2 r k}{(M-2 C) \sqrt{n \ln \ln n}} .
\end{gathered}
$$

Следовательно,

$$
X_{k+1} \geqslant M \sqrt{n \ln \ln n}-C \sqrt{(k+1) \ln \ln (k+1)}-\frac{2 r k}{(M-2 C) \sqrt{n \ln \ln n}},
$$

и остается показать, что при некотором выборе $M$ и всех $k<n$

$$
\frac{2 r k}{(M-2 C) \sqrt{n \ln \ln n}} \leqslant C \sqrt{(k+1) \ln \ln (k+1)}
$$

или

$$
\frac{4 r^{2}}{C^{2}(M-2 C)^{2}} k^{2} \leqslant(k+1) \ln \ln (k+1) \cdot n \ln \ln n .
$$

Так как $k<3(k+1) \ln \ln (k+1), k \geqslant 3$, и $k<n \ln \ln n$, это неравенство выполнено, если только значение $M$ выбрано достаточно большим. Лемма 1 доказана.

Из леммы 1 получаем:

$$
\mathbf{P}\left(B_{n} \mid A_{n}\right) \geqslant \mathbf{P}\left(B_{n} D \mid A_{n}\right)=\mathbf{P}(D)>0,
$$

откуда, согласно (14), выводится оценка

$$
\alpha(n) \geqslant \mathbf{P}\left(A_{n}\right)\left(\mathbf{P}\left(B_{n} \mid A_{n}\right)-\mathbf{P}\left(B_{n}\right)\right) \geqslant \mathbf{P}\left(A_{n}\right)(\mathbf{P}(D)-o(1)) \geqslant C(M \sqrt{n \ln \ln n})^{-\gamma},
$$

которая завершает доказательство теоремы 1 .

3 а м е ч а н и е 1. Заметим, что $\mathbf{P}\left(B_{n} \mid A_{n}\right) \geqslant \mathbf{P}(D)>0$ и $\mathbf{P}\left(B_{n}\right) \rightarrow 0$ при $n \rightarrow \infty$. Это влечет отсутствие $\varphi$-перемешивания, а результаты Ю.А. Давыдова и Р. Брэдли (см. [5]) приводят к заключению, что $\varphi(n) \equiv 1$.

3 а м е ч а н и е 2. Сравним нижнюю границу для $\alpha$-перемешивания и верхнюю границу для $\beta$-перемешивания. Существует зазор величины $1+\varepsilon$ между показателями в соответствующих оценках. В настоящее время автору неизвестно, появляется этот зазор в сил ${ }_{\lrcorner}^{r}$ различия между $\alpha$ - и $\beta$-перемешиванием или же это недостаток используемого метода. Ответ на этот вопрос может рассматриваться как интересное направление для дальнейших исследований. 
4. Субэкспоненциальная оценка. Модифицируя рассуждения в доказательстве теоремы 1 , можно получить нижнюю оценку в субэкспоненциальном случае.

Теорема 2. Для каждого $\varepsilon>0$ существует стационарная стохастическая модель, удовлетворяюшая предположениям предложения 2 , такая, что

$$
C_{1} \exp \left\{-c_{1} n^{\alpha /(1+p)}\right\} \leqslant \alpha(n) \leqslant \beta(n) \leqslant C_{2} \exp \left\{-c_{2} n^{\alpha /(1+p)-\varepsilon}\right\}
$$

с некоторьми константами $c_{1}, c_{2}, C_{1}, C_{2}>0$.

Д о к а з а т е л ь с т в о. Верхняя граница доказана в предложении 2. Рассмотрим случай $d=1$ и положим

$$
g(x)= \begin{cases}0, & x \leqslant R_{0} \\ x-r x^{-p}, & x>R_{0}\end{cases}
$$

где $r>0$ и $R_{0}>0$ имеют тот же смысл, что и в (6). При данном значении параметра $\alpha \in(0,1-p]$ найдем константу $B$, при которой

$$
B \int_{1}^{\infty} \exp \left\{-x^{\alpha}\right\} d x=1
$$

и положим

$$
\gamma=B \int_{1}^{\infty} x \exp \left\{-x^{\alpha}\right\} d x
$$

Предположим, что распределение случайных величин $V_{n}$ задается соотношением

$$
\mathbf{P}\left\{V_{n} \in A\right\}=\frac{1}{2} 1_{A}(-\gamma)+\frac{B}{2} \int_{A \cap[1, \infty)} \exp \left\{-x^{\alpha}\right\} d x .
$$

Элементарные вычисления показывают, что $\mathbf{E} V_{n}=0$ и $\mathbf{E} \exp \left\{\kappa\left|V_{n}\right|^{\alpha}\right\}<\infty$ для всех $\kappa<1$. Таким образом, предположение предложения 2 о существовании субэкспоненциальных моментов выполнено с $K=1$.

Точно так же, как и в полиномиальном случае, можно показать, что локальное условие Дёблина выполнено и инвариантная вероятностная мера сушествует и имеет атом в точке $-\gamma$.

Рассмотрим следуюшие события для стационарной версии процесса $\left(X_{n}\right)$ :

$$
A_{n}=\left\{X_{0}>M n^{1 /(1+p)}\right\}, \quad B_{n}=\left\{X_{n}>\frac{1}{2} M n^{1 /(1+p)}\right\}, \quad M>0 .
$$

Как и в полиномиальном случае, $\mathbf{P}\left(A_{n}\right) \rightarrow 0$ и $\mathbf{P}\left(B_{n}\right) \rightarrow 0$ при $n \rightarrow \infty$.

Используя соотношение

$$
\int_{M}^{\infty} \exp \left\{-x^{\alpha}\right\} d x \sim \alpha^{-1} M^{1-\alpha} \exp \left\{-M^{\alpha}\right\}, \quad M \rightarrow \infty
$$

оценим $\mathbf{P}\left(A_{n}\right)$ аналогично тому, как это было сделано в (14):

$$
\mathbf{P}\left(A_{n}\right) \geqslant c M^{1-\alpha} n^{(1-\alpha) /(1+p)} \exp \left\{-M^{\alpha} n^{\alpha /(1+p)}\right\}, \quad c>0 .
$$

По закону повторного логарифма для любой константы $C>0$ событие

$$
D=\bigcap_{k \geqslant 3}\left\{\sum_{j=1}^{k} V_{j} \geqslant-C k^{1 /(1+p)}\right\}
$$

имеет положительную вероятность. Возьмем $C=M / 4$.

Лемма 2. Пусть $n$ достаточно велико и события $A_{n}$ u $D$ произошли. Тогда для всех $1 \leqslant k \leqslant n$ события $\left\{X_{k} \geqslant M n^{1 /(1+p)}-2 C k^{1 /(1+p)}\right\}$ также произошли. 
Д ок а з а т е л с т в о. Используем индукцию. Предположим, что требуемые события произошли для $X_{0}, \ldots, X_{k}$. Представим $X_{k+1}$ как

$$
X_{k+1}=X_{0}+\sum_{j=1}^{k+1} V_{j}-r \sum_{j=0}^{k} \frac{1}{X_{j}^{p}} .
$$

Имеем

$$
r \sum_{j=0}^{k} \frac{1}{X_{j}^{p}} \leqslant \frac{r(k+1)}{(M-C)^{p} n^{p /(1+p)}}
$$

и

$$
\begin{aligned}
X_{k+1} & \geqslant M n^{1 /(1+p)}-C(k+1)^{1 /(1+p)}-\frac{r(k+1)}{(M-C)^{p} n^{p /(1+p)}} \\
& \geqslant M n^{1 /(1+p)}-2 C(k+1)^{1 /(1+p)}
\end{aligned}
$$

для достаточно большого $M$. Лемма 2 доказана.

Из этой леммы и (17) получаем точно так же, как и в полиномиальном случае, что

$$
\alpha(n) \geqslant C M^{1-\alpha} n^{(1-\alpha) /(1+p)} \exp \left\{-M^{\alpha} n^{\alpha /(1+p)}\right\} \geqslant C_{1} \exp \left\{-c_{1} n^{\alpha /(1+p)}\right\},
$$

и теорема 2 доказана.

3 а м е ч а н и е 3. Сравнив степени в субэкспонентах в оценках, полученных для $\alpha$ - и $\beta$-перемешивания, видим, что разница между ними может быть сделана сколь угодно малой. Следовательно, результат в [10] дает правильный показатель субэкспоненты и не может быть сушественно улучшен.

Автор признателен А.Ю. Веретенникову за детальное обсуждение вопроса, затронутого в статье.

\section{СПИСОК ЛИТЕРАТУРЫ}

1. Bradley R. C. Counterexamples to the central limit theorem under strong mixing conditions. - Colloq. Math. Soc. János Bolyai, 1984, v. 36, p. 153-172.

2. Bradley R. C. Basic properties of strong mixing conditions. - Progr. Probab. Statist., 1986 , v. 11 , p. $165-192$.

3. Bradley R. C. Identical mixing rates. - Probab. Theory Related Fields, 1987, v. 74, № 4, p. 497-503.

4. Bradley R. C. Counterexamples to the central limit theorem under strong mixing conditions. II. - Colloq. Math. Soc. János Bolyai, 1990, v. 57, p. 59-67.

5. Давыцов Ю. А. Условия перемешивания для цепей Маркова. - Теория вероятн. и ее примен., 1973, т. 18 , в. 2, с. 321-338.

6. Дуб Дж. Л. Вероятностные процессы. М.: ИЛ., 1956, 605 с.

7. Doukhan P., Massart P., Rio E. The functional central limit theorem for strongly mixing processes. - Ann. Inst. H. Poincaré, 1994, v. 30, № 1, p. 63-82.

8. Гринь А.Г. Предельные теоремы для схемы серий слабо зависимых величин. Теория вероятн. и ее примен., 1995, т. 40, в. 4, с. 888-897.

9. Ибрагимов И. А., Линник Ю. В. Независимые и стационарно связанные величины. М.: Наука, 1965, 524 c.

10. Klokov S.A., Veretennikov A. Yu. Sub-exponential mixing rate for a class of Markov chains. - Math. Commun., 2004, v. 9, № 1, p. 9-26.

11. Малыцихи М. Н. Субэкспоненциальные оценки скорости сходимости к инвариантной мере для стохастических дифференциальных уравнений. - Теория вероятн. и ее примен., 2000 , т. 45 , в. 3 , с. 489-504.

12. Merlevède F., Peligrad $M$. The functional central limit theorem under the strong mixing condition. - Ann. Probab., 2000, v. 28, № 3, p. 1336-1352.

13. Meyn S.P., Tweedie R.L. Markov Chains and Stochastic Stability. - London: Springer-Verlag, 1993, $548 \mathrm{p}$. 
14. Rosenblatt $M$. A central limit theorem and a strong mixing condition. - Proc. Natl. Acad. Sci. USA, 1956 , v. 42 , p. 43-47.

15. Веретенников А. Ю. Об оценках скорости перемешивания для стохастических уравнений. - Теория вероятн. и ее примен., 1987, т. 32, в. 2, с. 299-308.

16. Веретенников $А$. Ю. О полиномиальном перемешивании и скорости сходимости для стохастических дифференциальных и разностных уравнений. - Теория вероятн. и ее примен., 1999, т. 44 , в. 2, с. 312-327.

Поступила в редакцию

14.X.2002

Исправленный вариант

23.V.2005

(C) $2006 r$.

КУЗНЕЦОВА А. Я.*

\section{О СМЕСЯХ ОДНОВЕРШИННЫХ РАСПРЕДЕЛЕНИЙ}

В работе отмечаются некоторые полезные свойства смесей одновершинных распределений.

Ключевые слова и фразы: одновершинные распределения, смеси распределений, отклонение распределений от равномерного.

Мы ограничимся здесь специальным подклассом класса одновершинных распределений.

По определению, плотность вероятности $p(x ; X)$ случайной величины $X$ принадлежит классу CU (Continuous Unimodal), если она непрерывна и ограничена и существует такое $a$, что она не убывает при $x<a$ и не возрастает при $x>a$.

Имеет место следующее утверждение.

Лемма 1. Пусть $U-$ какая-либо случайная величина, плотность вероятности $p(x ; U)$ которой входит в класс CU. Тогда плотность вероятности $p(x ;\{U\})$ ее дробной части $\{U\}$ удовлетворяет неравенству: при любом $0 \leqslant x \leqslant 1$

$$
|p(x ;\{U\})-1| \leqslant 2 \max _{x} p(x ; U)
$$

(см. $[1$, пример $5, \S 8$, гл. II]).

Следствие 1. Пусть $\eta>0$ - масштабный параметр. Тогда в условиях леммы 1

$$
|p(x ;\{\eta U\})-1| \leqslant \frac{2}{\eta} \max p(x ; U) .
$$

Типичные примеры применения следствия 1 см. в [2] и [3].

Перейдем теперь к рассмотрению смесей (см. $[1, \S 5$, гл. II]) плотностей класса CU.

Пусть $U_{1}, \ldots, U_{k}$ - какие-либо случайные величины, плотность которых входит в класс $\mathrm{CU}$, и $p(x ; U)=\sum_{j=1}^{k} p\left(x ; U_{j}\right) \lambda_{j}$, где $\lambda_{j} \geqslant 0, \sum_{j=1}^{k} \lambda_{j}=1$.

Из следствия 1 с очевидностью вытекает

Следствие 2. При всех $0 \leqslant x \leqslant 1$

$$
|p(x ;\{\eta U\})-1| \leqslant \frac{2}{\eta} \sum_{j=1}^{k} \lambda_{j} \max p\left(x ; U_{j}\right) .
$$

* Московский государственный университет им. М.В. Ломоносова, факультет вычислительной математики и кибернетики, Ленинские горы, 119991 Москва, Россия. 